\title{
Quality of Life in Pediatric Patients with Serous Otitis Media using the 0M6 Questionnaire, Centro Médico Nacional Del Noroeste, Instituto Mexicano Del Seguro Social Sonora, México
}

\author{
Jade Espinosa ${ }^{1}$ and Juan Antonio Lugo Machado ${ }^{2 *}$ \\ ${ }^{1}$ Department of Otolaryngolgoy, Centro Médico Nacional del Noroeste, México \\ ${ }^{2}$ Surgeon Specialist in Otorhinolaryngology Department of Otolaryngolgoy, Centro Médico Nacional del Noroeste, México
}

*Corresponding author: Juan Antonio Lugo Machado, Surgeon Specialist in Otorhinolaryngology, Sub specialist in Pediatric Otolaryngology, Assigned Physician of Otolaryngology, National Medical Center of the Northwest, IMSS, Cd. Obregón, Sonora, Mexico.

Received Date: August 10, 2018

Published Date: August 29, 2018

\begin{abstract}
Background: To evaluate the quality of life in pediatric patients with serous otitis media using the OM6 questionnaire, in an Otorhinolaryngology service in Northwestern Mexico.

Introduction: Serous otitis media can affect balance, hearing and language development, causing poor school performance. This disease affects not only the child, but the whole family, from an economic and social point of view.

Material and methods: observational, retrospective, descriptive, transversal study. Full clinical records of the Otorhinolaryngology consultation in the northwest of the country were reviewed in the month of January 2017, ranging from 5 to 10 years old with a diagnosis of otitis media. The data were collected based on the OM6 survey. The variables of breastfeeding, exposure to cigarette smoke in the family nucleus, attendance at the nursery, family antecedent of WHO and schooling of the parents were also recorded.
\end{abstract}

Results: Of a total of 50 reviewed files. We found 39 complete clinical files. $69 \%$ were female and 31\% male. 51\% presented good quality of life and $49 \%$ presented poor quality of life. $36 \%$ had a history of exclusive breastfeeding $(\mathrm{p}<0.001)$.

Conclusion: There is a good quality of life in patients with a diagnosis of serous otitis media. A statistically significant association with breastfeeding was also found.

Keywords: Otitis media serosa; OM6 survey; Quality of life; Children

\section{Introduction}

Serous otitis media is simply the accumulation of fluid in the middle ear without signs and symptoms of ear infection. Asymptomatic serous otitis media can be classified as acute (less than 3 weeks), subacute ( 3 weeks to 3 months) or chronic (more than 3 months). The term acute or chronic refers to the time course of the disease, not the severity [1].

Serous otitis media is one of the most frequent diseases in the United States with more than 2.2 million cases diagnosed each year, with an annual cost of 4 billion dollars. [2] As many as $90 \%$ of children ( $80 \%$ individual ears) will have at least one episode of serous otitis media by the age of 10 years, with the majority of cases occurring between the age of 6 months to 4 years. Many episodes of serous otitis media resolve spontaneously within the first 3 months, but from 30 to $40 \%$ of children have recurrent episodes and 5 to $10 \%$ will last longer than 1 year [3].

Although rare, serous otitis media can also develop in adults, it usually occurs after a severe infection with upper areas, such as; acute rhinosinusitis, severe allergy or a rapid change in air pressure (barotrauma) after an airplane flight or a dive, the incidence of chronic serous otitis media in adults is unknown, but it is much less frequent than in the pediatric patient [4].

Some of the risk factors for serous otitis media include: male gender, recent upper airway infection, allergic rhinitis, first-degree relative with allergic rhinitis, bottle feeding, cigarette smoke at home, overcrowding and probably the most important factor is the daycare assistance. Additionally, some subpopulations of children 
are disproportionately affected by otitis media. Those with cleft palate, Down syndrome and other craniofacial anomalies are at high risk for anatomical reasons of suffering serous otitis media as well as inadequate function of the eustachian tube. [5]

The primary dysfunction of the eustachian tube results in decreased aeration and poor ventilation of the middle ear space; this results in a negative pressure with the consequent fluid transudation. Negative pressure in the middle hate also causes hypoxia and hypercapnia of the middle ear mucosa resulting in clear cell hyperplasia and hypersecretion. The result is a sterile fluid, which can be secondarily infected. [6]

The fluid resolves only when adequate ventilation of the middle ear has been restored, either by returning the proper functioning of the eustachian tube or by placing alternative ventilation, such as ventilation tubes [7].

Serous otitis media can be associated with discomfort and otic fullness. Patients with serous otitis media may be more prone to acute otitis media. The presence of transient hearing loss is common in patients with serous otitis media [8].

This hearing loss is often mild, but in some situations moderate or severe hearing loss may occur. Because this hearing loss can cause, in young children, a delay or a permanent change in their communication skills, resulting in a change in behavior and educational difficulties, so; There is genuine concern about the role of serous otitis media in these results [9].

In addition, those with chronic eustachian tube dysfunction, as well as otitis media, are at high risk of structural damage to the tympanic membrane [10].

Record an adequate clinical history to identify those patients at high risk of presenting serous otitis media. For example; It may be useful to inquire about recent upper respiratory infections, allergy, subjective or balance hearing loss, language delay or a history of craniofacial malformations [11].

Serous otitis media must first and then be distinguished from acute otitis media. Otitis media is diagnosed by the presence of fluid behind the tympanic membrane without the presence of acute signs of infection or inflammation. Acute otitis media, on the other hand, may include eustachian tube dysfunction and fluid in the middle ear, which must also include acute signs of infection and inflammation. Another fact that distinguishes between acute otitis media and otitis media serous; it is the appearance of the tympanic membrane, which in acute otitis media presents bulging and in serous otitis media it appears retracted or neutral [12].

In ear serous otitis, the tympanic membrane is opaque with hypomotility. In addition, hydro-aereo levels or bubbles can be appreciated. The use of pneumatic otoscopy to demonstrate decreased mobility of the tympanic membrane is considered a very important primary diagnostic method. Other factors necessary to confirm the diagnosis are; tympanometry, which will present a B (flat) curve, as well as a conductive type hearing loss in pure-tone audiometry [13].

Hearing is usually measured across the ranges of speech, and for young children normal hearing is considered no worse than $15 \mathrm{~dB}$ (which represents the necessary measure of intensity to respond to a sound). In contrast, the average hearing for ears with serous otitis media frequently reaches $25 \mathrm{~dB}$ and $20 \%$ exceeds $35 \mathrm{~dB}$. Although it is usually not necessary, diagnosis can also be made with imaging methods such as computed tomography of the temporal bone [14].

For children who do not present a risk of delay in language development, who are not considered to be at risk of presenting disorders in the development of learning, close observation of the evolution may be the therapeutic method of choice [15].

Hearing studies should be done if the middle ear effusion persists for more than 3 months or any time a disorder develops of language, difficulties in learning, there is a high clinical suspicion of decreased hearing threshold. If the average hearing is below $20 \mathrm{~dB}$, close observation is recommended, however if the best ear has an average hearing below $40 \mathrm{~dB}$, surgery is highly recommended. For children with hearing thresholds in the best ear between 21 to 39 $\mathrm{dB}$, management is based on the duration of the effusion and the severity of the symptoms [16].

The insertion of ventilation tubes is the most frequently performed ambulatory surgery in pediatric patients in the United States. The ventilation tube is placed in the tympanic membrane, to ventilate the middle ear cavity [17].

The risks and benefits of the placement of ventilation tubes in pediatric patients, has been analyzed by two systematic reviews recently. Juan Antonio Lugo Machado et al. [2] a Cochrane review of 10 randomized studies, concluded that the placement of ventilation tubes improves the short-term audiological result, but the benefit measure decreases from 6 to 9 months and does not present any difference of no differences seen in 12 to 18 months. With limited information, the effects of this on language development, cognitive development or impact on quality of life were not assessed [18].

A systematic review of 2011, commissioned by the Swedish Council for Health Technology Assessment, reviewed the ventilation tubes as the treatment for serous otitis media, without excluding special populations. Based on 8 randomized studies that followed the pediatric population for 10 years, the review concluded that there was strong evidence that ventilation tubes improve quality of life in a short period of time (nine months). The comparisons included; no treatment, observation and some other therapeutic methods such as decongestants and antihistamines [19].

Regarding the quality of life, Feagans and Blood described unclear language in children of 24 months with otitis media with effusion, [20] and Luloff found that these patients had predominantly problems in enunciating consonants [18]. While psychological tests measuring global intelligence have not shown that children with recurrent otitis are less intelligent, they have been found to be less persistent and attentive, and They show less enthusiasm in their responses in general [17].

The effect of previous or current serous otitis media on phonetic perception was examined in a study carried out by Pola and Rvachew when testing the discrimination of the goo and boo syllables in two test sessions in patients between the ages of 2 to 4 years. The function of the middle ear was assessed in both sessions 
by tympanometry. The result was divided into 3 groups; (a) negative history, children with normal middle ear function who have never received treatment for serous otitis media. (b) positive history, in children with normal middle ear function who have received medical treatment for previous episodes of serous otitis media. (c) children with serous otitis media who presented tympanometries with B curves on the day of the test [18].

It was found that children who had a negative history of serous otitis media performed significantly better than children in group $\mathrm{b}$ and group $\mathrm{c}$. These findings suggest that serous otitis media has a negative impact on the phonetic discrimination that may persist even after the function of the middle ear has returned to normal [21].

The quality of life as a result for the evaluation of treatment has become a recognized clinical entity and several studies have assessed the quality of life of children with serous otitis media. Since the development of the Otitis Media 6 (OM6) questionnaire in 1997, it has become the most applied questionnaire in the international literature. The questionnaire covers physical and emotional domains of the child's health status and concerns of the parent or guardian. Furthermore, the questionnaire includes a global measure of health status related to the child's quality of life. The questionnaire can be applied in a matter of minutes, making it excellent for application in different clinical scenarios [22].

To assess the impact on the quality of life of the pediatric patient with serous otitis media, several validated tools have been used. The Otitis Media 6 (OM6) survey is a disease-specific tool that validates 6 items reliably assessed by parents or guardians of children with serous otitis media. Represents the domains of physical suffering, hearing loss, alteration in speech or language development, emotional stress, limitation in the child's activities and the degree of concern of the parent or guardian [23].

In our study, the information of the medical notes of Otolaryngology was collected from the clinical file to answer the OM6 questionnaire of pediatric patients from 5 to 10 years old with a diagnosis of serous otitis media in the Specialties Hospital No. 2 of Cd. Obregón, Sonora, in order to know the quality of life in pediatric patients with said pathology.

\section{Methods}

Prior authorization by the local Ethics and Health Research Committee, an observational, descriptive, retrospective, crosssectional study was conducted in pediatric patients aged between 5 and 10 years entitled IMSS, diagnosed with serous otitis media by a specialist in otolaryngology in the period from April 1 to September 30, Juan Antonio Lugo Machado et al. [2] at the National Medical Center of the Northwest, IMSS, Cd. Obregón, Sonora, Mexico which meet selection criteria. The sample consisted of pediatric patients selected by non-probabilistic sampling whose clinical records are available for the Otorhinolaryngology service. Once identified by the daily record of consultation those patients diagnosed with serous otitis media during the month of January 2017. The files were obtained in the hospital's clinical file, based on the medical note of the Otorhinolaryngology consultation. the information necessary to complete the survey already validated 0M-6. Which was built by Rosenfeld, et al.4 and later validated by Timmermman et al. [19] and which is applicable to patients between 6 months and 12 years, this survey evaluates 6 dimensions: physical suffering, hearing loss, limitation of language, emotional problems, limitation of activities, concern of the parent or caregiver. The data obtained was concentrated in an individual format which contained $100 \%$ of the variables to be measured, subsequently;

The data were recorded in special sheets and recorded in a database for tabulation and analysis. The results obtained from the tabulation of these instruments were organized, collected, and entered into the database. A descriptive-type statistical instrument was applied with measures of central tendency for qualitative variables by means of frequencies and percentages. Associations were established through the use of the odds ratio and Spearman's Rho, a 2 × 2 contingency table and 95\% confidence intervals were made. The significance

The statistics of these results were analyzed using Pearson's Chi square test for qualitative variables. The results were represented in graphs, tables and/or tables and described according to the type of information. A value of $\mathrm{p} \leq 0.05$ was considered significant. The statistical package SPSS version 22 for Windows ${ }^{\circledR}$ was used to perform the respective statistical analysis.

\section{Results}

When taking into account the average score of the survey OM6 registered a tendency to present better quality directly proportional with age. In the 5-year-old patients, the average score was 4.11 , while in the 10-year-old patients it was 2.43 (Table $1 \& 2$ )

Table 1: Average age of the patients studied.

\begin{tabular}{|c|c|c|c|}
\hline Minimum & Maxima & Media & $\begin{array}{c}\text { Standard } \\
\text { deviation }\end{array}$ \\
\hline 5 & 10 & 7.28 & 1.7 \\
\hline
\end{tabular}

Table 2: Average OM6 by age and DS.

\begin{tabular}{|c|c|c|}
\hline Age & Average score & SD \\
\hline 5 & 4.117 & 0.96307 \\
\hline 6 & 3.75 & 0.32031 \\
\hline 7 & 3.4057 & 1.10496 \\
\hline 8 & 2.8617 & 0.70312 \\
\hline 9 & 3.1671 & 1.08329 \\
\hline 10 & 2.436 & 0.69374 \\
\hline
\end{tabular}

Table 3: Variable correlation.

\begin{tabular}{|c|c|c|}
\hline & OM-6 & $\mathbf{p}$ \\
\hline Gender & -0.094 & 0.569 \\
\hline Kindergarten & -0.306 & 0.062 \\
\hline Smoking & -0.291 & 0.072 \\
\hline Breastfeeding & 0.494 & 0.001 \\
\hline Antecedents or more & -0.021 & 0.897 \\
\hline Scholarship & 0.545 & 0 \\
\hline
\end{tabular}

It was also found that $25(64.8 \%)$ children attended day care, and 14 (35.9\%) did not attend day care. Being in daycare presented a relative risk of 2.37 times more to have poor quality of 
life compared to not having attended daycare, was not statistically significant ( $p=0.062)$. (Table 3); $14(35.9 \%)$ of the patients had a history of exclusive breastfeeding, 14 (35.9\%) patients had bottle feeding and 11 (28.2\%) mixed feeding, with a relative risk of 0.259 ( $\mathrm{p}<0.001)$. (Table 3 and chart 2); $16(41 \%)$ of the patients had a positive first-degree family history of presenting episodes of WHO in childhood and 23 (59\%) had no history. The family history of otitis media had no impact on a poor quality of life $(R R=1.030, p=$ 0.089) (Table 1 \& 2)

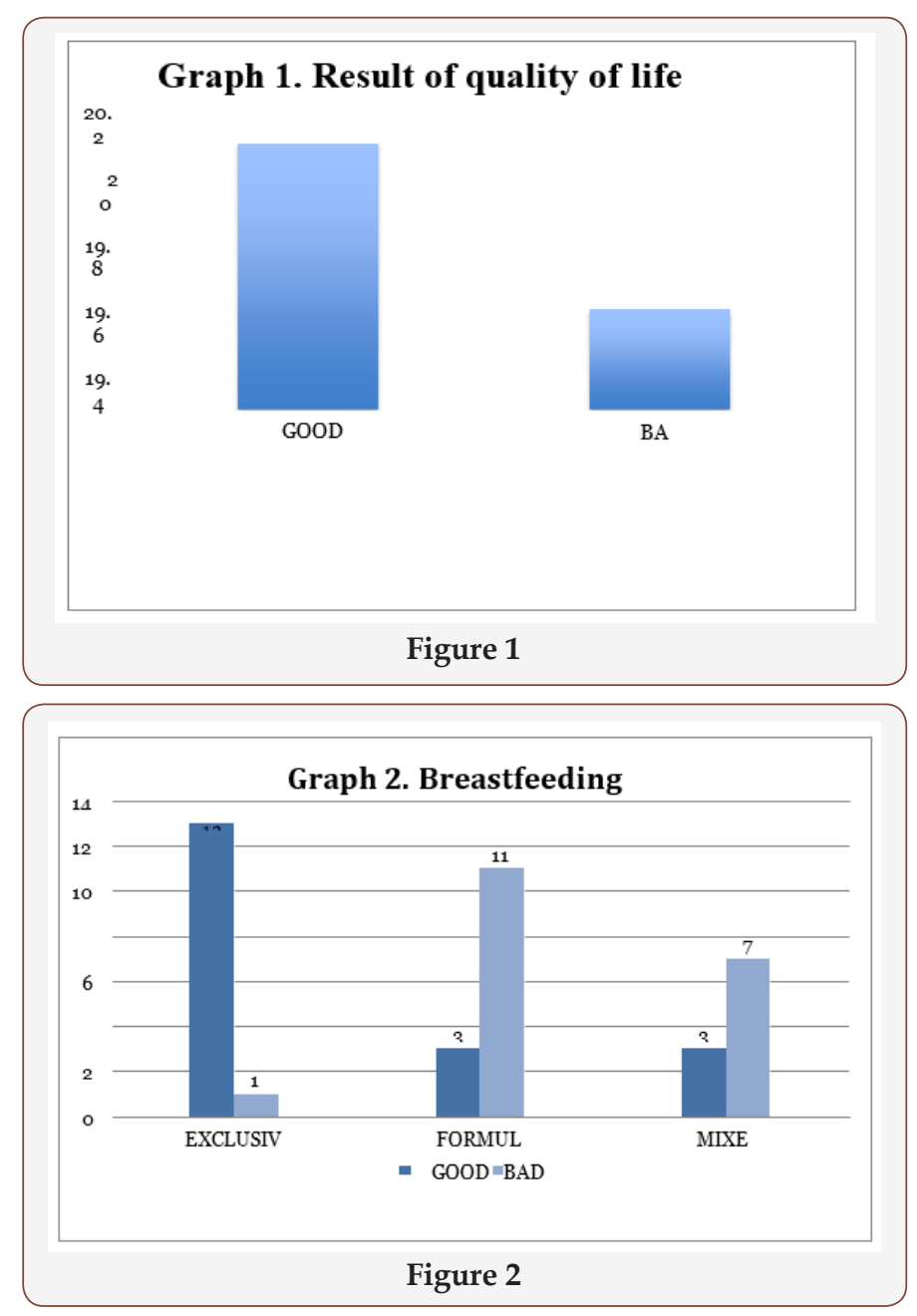

In $6(15.4 \%)$ of the children, their parents had primary school level education, 13 (33.3\%) had secondary school, 11 (28.2\%) had a baccalaureate and 9 (23.1\%) had a bachelor's degree. Comparing the schooling of the parents with the quality of life, it was found that there is a relative risk of 2.66 times greater than having a poor quality of life if the parents have higher or higher average schooling ( $p=0.007$ ) (Figure 1-3).

In $17(43.6 \%)$ patients were exposed to smokers in the family environment; 22 (56.4\%) were not exposed to smokers in the family environment. Being in an environment with smoking relatives presented a relative risk of 1.66 times more to have poor quality of life $(\mathrm{p}=0.08)$.

From here it is emphasized that the main risk factor of those studied for a poor quality of life is the superior schooling of the parents. The associated protective principal factor turned out to be breastfeeding covariates except for "methods available for error detection." Note that not every hospital participated in MEDMARX for all five years (i.e., from 2003 to 2007), although the proportion of hospitals utilizing CPOE grew from $38.1 \%$ in 2003 to $50.8 \%$ in 2007. Unlike CPOE use, the proportion of healthcare facility with computer-generated medication administration records (MARs) did not vary significantly.

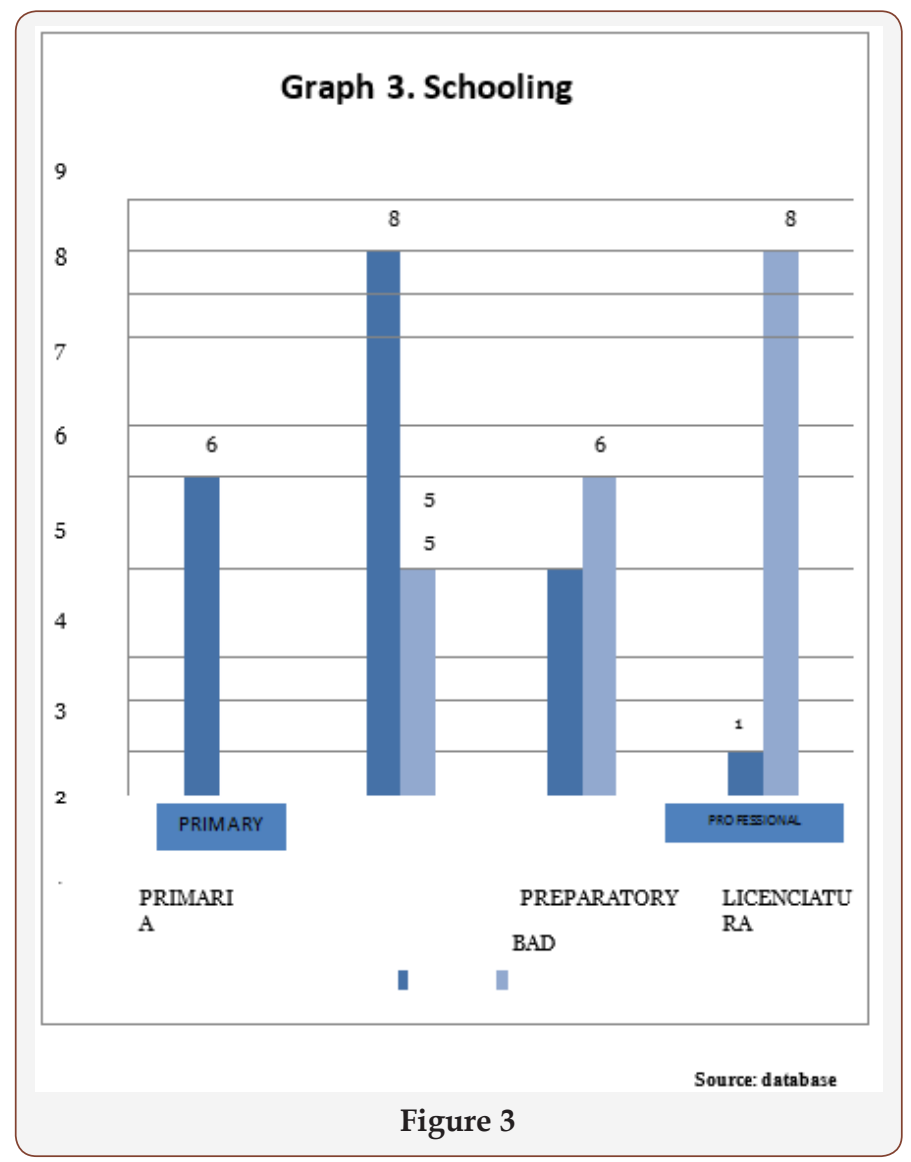

\section{Discussion}

In this study we sought to demonstrate how the quality of life of these patients is not affected and the variables that could be influencing these results. The average scores found coincide with other reports of international studies published by Rosenfeld et al., 4 however; the author conducted a study with a larger sample of patients, which enhances the quality of this study. In the previously mentioned study, the simple reproducibility of the application of the instrument is mentioned, which was corroborated in our study.

Another very interesting study is Haytham et al. [2] in which the Danish author found certain flaws with the instrument in terms of parental appreciation about the domains of hearing loss and language problems. This may explain in part the reason why most patients presented a better quality of life, because it is directly related to the interpretation of the parents and their sociocultural level.

In a multicentric study by Witsell et al. [11] where the result of the placement of ventilation tubes is assessed, by applying the 0M-6 survey, it obtains without replacement; results contrary to those obtained in our study, since in this study they show an improvement in the score obtained after surgical treatment. Which can be talking about an underestimation of the problem by the 
parent or guardian, same result that may be the one that has been obtained in this study, since there was no control after the surgical treatment.

It is possible to relate, through the evaluated variables, some of the factors that could be influencing the result of the scores of our patients. It is widely described in the literature of Otorhinolaryngology, the factors that are associated with the presentation of upper airway infections, including within these to serous otitis media. However, until now an evaluation of the quality of life and the factors that predispose to serous otitis media have not been carried out. A correlation to analyze in depth what it was the academic degree of the parents and the quality of life, given that the survey is answered by them, it may be that the higher the level of education, the more they perceive the condition towards their child and therefore; the result obtained Within our greatest limitation was the size of the sample, which due to lack of time could not be extended, giving a greater validity to the study. We propose the realization of a prospective study that includes the carrying out of the 0M-6 survey before and after the surgical treatment.

\section{Conclusion}

The quality of life of pediatric patients between 5 and 10 years old with a diagnosis of serous otitis media in the northwest of the country is good.

There are statistically significant differences between breastfeeding and its association with an OM-6 result with a good quality of life.

\section{References}

1. Cassel Brandt LM (2010) Acute otitis media and otitis media with fussion. Flint WP (Ed.), Cummings otolaryngology head and neck surgery fifth edition. Estados Unidos, Mosby-Elsevier, Netherlands, pp. 2761-2777.

2. Haytham K, Gatehouse S, Swan IR (2004) How appropriate is the 0M-6 as a iscriminative instrument in children with otitis media? Arch Otolaryngol Head Neck Surg 130(6):705-709.

3. Kesser WB, Derebery JM, Friedman RA (2010) Surgery of Ventilation and mucosal disease. Brackman S (Ed.), Otologic surgery. ( $3^{\text {rd }}$ edn), Estados Unidos, Saunders-Elsevier, Netherlands, pp. 73-89.

4. Rosenfeld RM, Goldsmith AJ, Tetlus L, Balzano A (1997) Quality of life for children with otitis media. Arch Otolaryngol Head Neck Surg 123(10): 1049-1054.

5. Rosenfeld RM, Bhaya MH, Bower CM, Brookhouser PE, Casselbrant ML, et al. (2000) Impact of tympanostomy tubes on child quality of life. Arch Otolaryngol Head Neck Surg. 126(5): 585-592.

6. (2008) National Collaborating Centre for Women's and Children's Health. Surgical Management of Otitis Media With Effusion in Children. National Institute for Health and Clinical Excellence, London, UK.

7. Rosenfeld RM, Goldsmith AJ, Madell JR (1998) How accurate is parent rating of hearing for children with otitis media? Arch Otolaryngol Head Neck Surg 124: 989-992.
8. Boruk M, Lee P, Faynzilbert Y, Rosenfeld RM (2007) Caregiver well-being and child quality of life. Otolaryngol Head Neck Surg 136(2): 159-168.

9. Berkman ND, Wallace IF, Steiner MJ, Harrison M, Greenblatt AM, et al. (2013) Otitis Media with Effusion: Comparative Effectiveness of Treatments.

10. Lee J, Witsell DL, Dolor RJ, Stinnett S, Hannley M (2006) Quality of life of patients with otitis media and caregivers: a multicenter study. Laryngoscope 116(10): 1798-1804.

11. Witsell DL, Stewart MG, Monsell EM, Hadley JA, Terrell JE, et al. (2005) The Cooperative Outcomes Group for ENT: a multicenter prospective cohort study on the outcomes of tympanostomy tubes for children with otitis media. Otolaryngol Head Neck Surg 132(2): 180-188.

12. Rovers MM, Krabbe PF, Straatman H, Ingels K, van der Wilt GJ, et al. (2001) Randomised controlled trial of the effect of ventilation tubes (grommets) on quality of life at age 1-2 years. Arch Dis Child. 84(1): 4549.

13. Brouwer CN, Maille AR, Rovers MM, Grobee DE, Sanders EA, et al. (2005) Health-related quality of life in children with otitis media. Int J Pediatr Otorhinolaryngol 69(8):1031-1041.

14. Rosenfeld RM (2005) A parent's guide to Ear tubes. Hamilton, Canada.

15. Rosenfeld RM, Culpepper L, Doyle KL, Shin JJ, Schwartz SR, et al. (2004) Clinical practice Guideline: Otitis media with efussion. Otolaryngol Head and Neck Surgery 130(5 suppl): S95-S118.

16. Mui S, Rasgon BM, Hilsinger RL, Lewis B, Lactao G (2005) Tympanostomy tubes for otitis media: quality of life improvement in children and their parents. Ear Nose and Throat J 84(7): 418-420.

17. Feagans LV, Blood IM (1993) Language and behavioral sequelae of otitis mediain infants and young children attending day care centers. Lim DJ, Bluestone CD, Klein JO, Nelson JD, Ogra PL, (Eds.), Recent advances in otitis media. Proceedings of the Fifth International Symposium. Hamilton, Decker Periodicals, USA, pp. 521-523.

18. Moore DR, Hutchings ME, Meyer SE (1992) Binaural masking level differences in children with a history of otitis media. Hear Res 63: 71-78.

19. Polka L, Rvachew S (2005) The impact of otitis media with effusion on infant phonetic perception. The official journal of the international congress on infant studies.

20. Heidemann C, Godballe C, Kjeldsen AD, Johansen EC, Faber CE, et al. (2013) The otitis media-6 questionnare: psychometric properties with emphasis on factor structure and interpretability. Health and quality of life outcomes.11:201.

21. Marchisio P, Bellussi L, Di Mauro G, Doria M, Felisati G et al. (2010) Acute otitis media: From diagnosis to prevention. Summary of the Italian guideline. Internat J Pediatr Otorhinolaryngol 74: 1209-1216.

22. Vergison A, Dagan R, Arguedas A, Bonhoeffer J, Cohen R, et al. (2010) Otitis media and its consequences: beyond the earache. Lancet Infect Dis 10(3): 195-203.

23. Timmerman AA. Anteunis LJ, Meesters CM (2003) Response-shift bias and parent-reported quality of life children with otitis media. Arch Otolaryngol Head Neck Surg 129(9):987-991. 